\title{
Vertical Rectus Abdominis Musculocutaneous Flap Repair Improves Perineal Wound Healing After Abdominoperineal Resection for Irradiated Locally Advanced Rectal Cancer
}

\author{
Samuel Levy, MD, Kevin Serror, MD, and David Boccara, MD, PhD \\ Service de Chirurgie Plastique, Reconstructrice Et Esthétique, Centre des Brûles, Hopital Saint-Louis, Paris, France
}

\section{TO THE EDITOR}

We read with interest the manuscript by Spasojevic et al. ${ }^{1}$ entitled Vertical Rectus Abdominis Musculocutaneous Flap Repair Improves Perineal Wound Healing After Abdominoperineal Resection for Irradiated Locally Advanced Rectal Cancer. Their goal was to determine whether perineal wound healing was better after direct wound closure than with a vertical rectus abdominis musculocutaneous (VRAM) flap repair.

Spasojevic et al. concluded that patients with direct wound closure had less likelihood of perineal wound healing after 3 months, with a number needed to treat of five patients to prevent one delayed wound healing. Nevertheless, they found no difference between the two groups in terms of the rate of severe complications and perineal wound complications. These results led them to conclude that the VRAM flap procedure should only be reserved for selected patients.

We wished to share our experience of the 'Paris Protocol' that has been previously described., ${ }^{2,3}$ Indeed, we compared our 104 patients who were operated with a VRAM flap after abdominoperineal resection, between 2000 and 2016, with those of the non-VRAM group in the study by Spasojevic et al. We focused on age, gender, comorbidities, and postoperative morbidity, and used the Chi square test to compare variables. We found no difference regarding comorbidities and medical history, with the exception of gender. However, unlike Spasojevic et al., we found a significant diminution of 30-day severe complications $(13.5 \%$ of patients; $p<0.0001)$ and perineal wound complications $(22.1 \%$ of patients; $p<0.01)$. The reasons for early reoperation were abscess or partial necrosis requiring debridement (five cases), occlusion (four cases), flap loss (three cases), stoma repair (one case), and parietal hernia (one case).

We believe that these results should be taken into account because they may favor the VRAM flap closure procedure. Indeed, we believe that this procedure becomes safer and safer with the standardization of postoperative care. ${ }^{4}$ It enhances perineal wound healing security and provides a better quality of life. For these reasons, we use VRAM flap closure on all our patients who require an abdominoperineal resection.

\section{REFERENCES}

1. Spasojevic M, Mariathasan AB, Goscinski M, et al. Vertical rectus abdominis musculocutaneous flap repair improves perineal wound healing after abdominoperineal resection for irradiated locally advanced rectal cancer. Ann Surg Oncol. 2018;25(5):1357-1365.

2. Boccara D, Serro RK, Lefevre J, Mimoun M, Chaouat M (2017) Reconstruction par lambeau de Taylor après amputation abdomino-périnéale: à propos de 68 cas. Ann Chir Plast Esthét. https://doi.org/10.1016/j.anplas.2017.09.009

3. Boccara D, Serror K, Levy S, Mimoun M, Chaouat M. Postoperative care of VRAM flaps for perineal reconstruction. J Plast Reconstr Aesthetic Surg. 2018;71(3):446-447.

4. Warbrick-Smith J, Drew PJ. Post-operative care of VRAM flaps for perineal reconstruction: results of a UK practice survey and literature review. J Plast Reconstr Aesthetic Surg. 2018;71(2):271-273.
(C) Society of Surgical Oncology 2018

First Received: 23 March 2018;

Published Online: 19 June 2018

S. Levy, MD

e-mail: samtlevy@gmail.com 